\title{
Policy Challenges of Electronic Health Records and Meaningful Use
}

\section{David Randalı}

American Research and Policy Institute, Washington, DC, USA

The promise of universal provider adoption of electronic records systems has been a 'holy grail' for the health information technology market. Recent industry and government reports suggest that adoption of electronic health record standards outlined by the 2009 law that has funded much of the industry growth is lagging behind with a good deal of variation in provider adoption rates. These facts take on added significant as the Centers for Medicare and Medicaid Services (CMS) tie future funding to provider adoption and 'meaningful use' attestation by providers.Given these coming milestones and varying degrees of implementation success, serious questions remain about the future of wide-spread adoption of electronic health records systems and how the U.S healthcare system will look in the coming decade.

The U.S. Recovery Act of 2009 and the accompanying HITECH Act of 2009 provided an enormousfinancial boost toward adoption of electronichealth records with over $\$ 34$ Billion appropriated to fund provider information technology hardware and software solutions [1]. As implementation has progressed, there are numerous milestones established by both the authorizing statute, but also under CMS guidelines and regulations. The so called 'meaningful use' guidelines are set in series of stages, with Stage 1 designed to facilitate data capture and sharing, which ended in 2012. Stage 2 of the program is intended to advance clinical processes for all eligible healthcare professionals and hospitals and to report to CMS by the end of 2014.The final Stage 3 of the program is slated to be fully implemented by 2016 with goal of improvedclinicaloutcomes as result of EHR use by providers [2]. Each stage of the implementation process has had varying degrees of success with both hospitals and provider professionals as evidenced by both published research and various industry reports. The variation in adoption rates of EHRstandards raises the greatest levels of concern among health IT professionals, policy makers and academics about the prospects for wide adoption of uniform electronicstandards.

Adoption rates and the progress toward Stage 3 EHR standards continue to be of concern to not only providers, but policymakers as well.Recent reports suggest that while EHR use has grown from $20 \%$ of providers using the technology in 2002 to over $60 \%$ today, much of the increased use has been uneven across professional providers and hospitals [3]. Rural hospitals have generally lagged their peers and older physicians (greater than age 55) have lower adoption rates [4]. The lack of adoption success among all provider types can be attributed to many factors other than the structure of the current CMS incentive systems. Other evidence and previous research suggests that achieving 'meaningful use' also has to do with patient populations, clinical outcomes, and private and public payer reimbursement methodologies $[5,6]$. Given these lower than expected adoption rates, many health IT firms have struggled to achieve CMS guidelines with their clients as many small providers struggle to implement Stage 2 standards, which leaves serious questions about how the majority of professional providers can achieve Stage 3 attestation and meaningful use standards.

Federal government agencies are not immune to the challenges of implementing EHR standards.Both the Veteran's Administration and the Department of Defense have had numerous implementation issues in their attempt to use EHR technology and to increase the interoperability of their respective systems [7]. With billions being spent on IT systems, software, consultants and hardware at federal agencies with massive IT procurement budgets, a natural question is what hopes do small hospitals and solo health care professionals have in meeting Stage 3 standards? Recent reports confirm that CMS has acknowledged the failure of many smaller providers and group physician practices to achieve the initial standards and CMS has given 'additional flexibility' to meet Stage 2 and Stage 3 attestation [8]. The failures at these federal agencies suggest that many challenges remain in achieving not only universal technology use of electronic health records but also the anticipated success of Stage 3 guidelines.

As policymakers and health care providers struggle with Stage 2 and Stage 3 attestations, the future of wide adoption of EHR standards remain a looming policy dilemma. Our recent research about the potential effect of insurer payment lags on electronic medical records use suggests that additional incentives may be an effective policy tool to spur adoption. We suggest the Federal Government should begin tackling the how of payment reform through the Federal Employee Health Benefits Plans (FEHBP), since that would allow the federal government to provide a substantial incentive for EHR adoption by mandating payment reform in the areas of common provider identification numbers, online insurance verification, payment assurance, and all electronic-transactions [9]. Utilizing existing government systems to test the above incentives is practical and incremental policy choice to potentially spur greater adoption rates among all provider types, including smaller hospital systems and physician group practices that have recently struggled to meet the guidelines.

Insurers and public payers must take a much more active role in spurring adoption rates if the healthcare systems efficiencies envisioned by EHR adoption can be achieved in the next decade. Given the recent reports, it is unlikely that the Stage 3 attestations will achieve the desired results that CMS and other policy observers had hoped.In short, incentive methods tied to payment systems should be explored more rigorously across all payers in order achieve the efficiencies that can improve the U.S healthcare delivery system.

\section{References}

1. American Recovery and Reinvestment Act of 2009, Public Law 111-5, Title XIII.

2. Centers for Medicare and Medicaid Services "EHR and Meaningful Use definitions and standards

3. Murhpy K (2012) EHR Intelligence: EHR adoption depends on age group, practice size and setting, and specialty".

*Corresponding author: David Randall, American Research and Policy Institute Suite 200, 1250 Connecticut Avenue, NW, Washington, DC, 20036, USA, Tel: 202558-6364; Fax:202-446-2964; E-mail: info@arapi.org

Received August 28, 2014; Accepted September 24, 2014; Published October 01,2014

Citation: Randall D (2014) Policy Challenges of Electronic Health Records and Meaningful Use. J Health Med Informat 5: 165. doi:10.4172/2157-7420.1000165

Copyright: (c) 2014 Randall D. This is an open-access article distributed under the terms of the Creative Commons Attribution License, which permits unrestricted use, distribution, and reproduction in any medium, provided the original author and source are credited. 
Citation: Randall D (2014) Policy Challenges of Electronic Health Records and Meaningful Use. J Health Med Informat 5: 165. doi:10.4172/21577420.1000165

Page 2 of 2

4. Decker S, Jamoon E, Sisk J (2012) Physicians In Non primary Care And Small Practices And Those Age 55 And Older Lag In Adopting Electronic Health Record Systems. Health Aff31:1108-1114.

5. Adler-Milstein J, Kvedar J, Bate DW (2014) Telehealth among US hospitals: several factors, including state reimbursement and licensure policies, influence adoption. Health Aff33: 207-215.

6. Ash JS, Bates D (2005) Factors and forces affecting EHR system adoption: report of a 2004 ACMI discussion. J Am Med Inform Assoc12: 8-12.
7. Government Health IT News. "Congress demands answers to VA, DoD interoperability progress".

8. US Department of Health and Human Services. HHS provides additional flexibility for certification of electronic health.

9. Ramlet MJ, Randall D, Parente ST (2013) Insurer Payment Lags to Physician Practices: An Opportunity to Finance Electronic Medical Record Adoption. J Health Med Informat 4: 136. 